\title{
A.JO'ГE
}

African Journal of Teacher Education

ISSN 1916-7822. A Journal of Spread Corporation

Volume $92020 \quad$ Pages $70-79$

\section{Accent and Ugandan Students' Comprehension of Mathematical Concepts and Terms: An Experimental Study.}

\author{
Imelda Kemeza ${ }^{1}$, Sudi Balimuttajjo \\ Mbarara University of Science and Technology, Uganda \\ and \\ Dinesh G. Sarvate \\ College of Charleston, \\ USA
}

\begin{abstract}
:
The embrace of diversity and multiculturalism in education facilitates the broadening of students' experiences as they engage with teachers and classmates from backgrounds different than their own. However, while the positive effects of diversity on students are apparent, few studies have examined possible negative challenges that diversity might have on students. Where most subject matter is taught via classroom lectures and the lecture material is presented by a speaker with a different accent than the student is used to hearing, does it make the material harder for the student to understand? On the other hand, could it increase the focus and engagement required by the students in the classroom, and in the process increase their understanding? In this vein, our research sought understand whether students' learning of the subject matter hindered when they are taught material by a teacher with a different accent. To this end, we designed a simple experiment with a small group of undergraduate students in Uganda, to address this question, the result of which we present in this study.
\end{abstract}

Keyword: accent, English speaker, comprehension, mathematical concepts, Uganda.

\section{Introduction:}

In today's world of shrinking global distances, increasing human migration across countries and continents, and increasing global trade, it is inevitable that students will come across teachers who are completely different from them by race, culture, mannerism, or accent. It has been noted that

\footnotetext{
${ }^{1}$ Contact: Imelda Kemeza. Email: ikemeza@must.ac.ug
} 
even though the instruction itself may be given in a familiar language like English, hurdles still exist. Students might perceive foreign teachers' accents as strange and incomprehensible (Kolapo, 2009). In addition, it is not only the accent but also the syntactic structure that an English speaker uses that may affect student learning. It is the authors' experience that for most students, learning usually gets better as time passes and students get used to the teachers' accent. However, there are very few, if any, studies that highlight the issue of accent in the classroom and its impact on learning outcomes in institutions of higher learning in Uganda. Therefore, we wish to examine accent as an important communication hindrance to learning in mathematics.

Uganda has 40 known living indigenous /native languages. A group of native speakers who keep in close contact grow to pronounce the same words differently from other groups of speakers of the same language and are said to speak the same language with different accents. Uganda languages are grouped into three language families; Bantu (examples include, Nyoro, Tooro, and Runyankole), Central Sudanic (or Nilo-Saharan, examples, including, Aringa, Lugbara, and Pokot), and Nilotic (examples include Acholi and Alur. The Central Sudanic language family comprises of 60 languages, the Nilotic 29 and Bantu 12 languages. Mbarara University of Science and Technology, the locus of this study, admits students from across these language families.

Variation in pronunciation of words based on different accents implies that it is easier for students to understand the spoken English of those who pronounce the same words as themselves. Some accents substitute sounds, syllables, and letters making for significant variations to the tone, pitch, and the sounds of words; for example, $\mathrm{L}$ is substituted for $\mathrm{R}$ and others substitute ch for $\mathrm{S}$ and yet others elide some syllables that others sound out. This accent variation affects how they pronounce and comprehend English words during their education from elementary through secondary school. A non-Uganda speaker of English thus introduces significant variations to the pronunciation and understanding of English words.

Most often, teachers of mathematics, including in Uganda, expose students to subject content through expositions on the white/blackboard. Alongside board work, teachers of mathematics help students solve problems through explanations to become more successful in targeted mathematics areas (Gurel Cennetkusu, Koc, \& Teker Kozcaz, 2020). Typically, as teachers solve problems on the boards, they are equally teaching how to solve similar problems in other topic areas on the board, i.e., transfer of learning with help of analogues. Of the two teaching methods, accent differences likely 
affect students' comprehension of board work less than with oral explanations where there is a high probability of accent domination.

\section{Theoretical Background}

A teacher's accent can have direct and indirect effects on learning activities. The difficulty is twofold; the student's inability to comprehend the lecturer's accent as well as the second problem of the consequent hindrance to understanding the subject content (Park, Klieve, Tsurutani \& Harte, 2017; Sicat, 2011; Campbell \& Li, 2008). Multiple studies have investigated the effects of English as a second language on comprehensibility using rating scales and methodologies (Gurel Cennetkusu, Koc, \& Teker Kozcaz, 2020; Hou, 2018; Valles, 2015; \& Lippi-Green, 2012). However, only a few studies have investigated students understanding in mathematics when English is used as a medium of instruction in the course of verbal exchange through questions, instructions and explanations between students and lecturers.

An early study by Taussky (1970) posits that when students are introduced to mathematical terms, concepts, and information, (like theorems about the sum of the squares that we used in the current study), the chances are that the students comprehend the functionality of integers or prime numbers but not the theorems underlying them, such as expressing them as sums of different number of squares. In this study, apart from having prior knowledge of sums of squares in the Pythagoras theorem, students had no access to the pre-recorded material and the quiz that was used in the study.

Comprehension is the ability to understand the meaning of an action or concept. In theory as well as in practice, comprehension is seen as the basis for quality teaching and learning (Smith, 1975). This is crucial because the ways students comprehend squares of integers is the foundation for all their further interaction with squares of various numbers. To achieve this, however, students must invest some mental effort in quality learning (Salomon, 1983) in a setting where the teaching is clear, bold, and connected to the context (Keene \& Zimmermann, 1997).

A difference in accent between teachers and students may pose an obstacle to achieving the goal of clear teaching, especially in mathematics instruction. Comprehension of mathematical concepts requires students to become familiar with words whose mathematical meaning is different from their general and commonly associated meanings. For example, take the word "prime", wherein common language it means "first" as in "prime minister" or "main" as in "prime suspect", in math it 
takes on a whole new meaning. To progress in math, irrespective of their own dialects and accents, students must deduce meaning from the speaker's oral explanation in order to construct an understanding of the mathematical concepts involved in the seemingly complicated mix of information in phrases such as:

"any positive integer can be expressed as the sum of at most 4 squares"

"by exactly two if a prime, giving a remainder of 1 when divided by 4 "

"if the remainder is 3 , then the prime can be expressed as the sum of three squares"

Problem-solving is an integral part of mathematics education. It is critical for students to decipher the true meaning of words when they listen to verbal descriptions of mathematical concepts. In this study, we test students' ability to understand oral mathematical examples, which understanding they can then successfully use to solve problems on a quiz.

\section{Aim and questions addressed}

The aim of the study was to assess the impact of a teacher's accent on a learner's comprehension of mathematical concepts and terms.

\section{Method and Description of the experiment}

In trying to determine the effect of accent on the understanding of mathematical terms and concepts, we designed an experiment strictly about learning mathematical concepts with the only variable being what accent was used in presenting the material. A script was written demonstrating a math concept. This script was independently read verbatim by Ugandan and non-Ugandan English speaking instructors who were recorded on tape. A neutral person then played back the pre-recorded material to students who were asked to listen to the material and answer in writing some questions based on the materials to which they just listened. By eliminating the visual presence of the teachers, we were able to control for the individual mannerism and language structure of the teachers, and also remove any preconceived ideas or hesitation on the part of the students that might arise when they initially see a teacher in person. In addition, we wanted to avoid the visual and written forms of communication that board work introduces. We chose our topic of study such that we could ask students to solve problems without having to explain solutions using board work or any practice problems. In so doing we directly avoided the effect of teacher's written communication on students' understanding and were able to isolate just the effects of verbal communication. 


\section{Participants}

Second- and third-year Ugandan undergraduate university students were purposely recruited. They were all volunteers and had no prior knowledge of the content in the mathematics passage. Only students majoring in mathematics were eligible to participate in the study; students majoring in Chemistry, Physics and Biology but with mathematics as an elective were excluded. For the study, two different recorded readings, one by an English speaker with a native accent and the other by a non-Ugandan English speaker with a foreign accent, were played to two different sets of students. Each set of students was asked to listen to the recording with the goal of understanding the material and then was given a quiz on the information presented. The procedure was as follow:

- A recorded mathematical passage of a non-Ugandan English speaker accent was played to the first set of selected Ugandan undergraduate students. The students spoke any of the three main Ugandan language families as their first language but routinely received instruction in English which is their second language.

- A recording of the same mathematical passage by an English speaker with a native Ugandan accent was played to the other set of Ugandan undergraduate students. These students also spoke any of the main three language families in Uganda as their first language, but also routinely received instruction in English which is their second language.

- After listening to the passage, the students answered questions from a quiz on the topic.

English is the medium of instruction in Uganda and is used as a second language by all the students who participated in the study.

\section{Variables and measures}

\section{Instrument}

The two speakers employed to pre-record the mathematics instruction for this experiment, the first with a non-Ugandan accent, and the other with a native Ugandan accent, both learned English as a second language. Both of them speak English with clearly different accents and consequently their pronunciation of mathematical terms and concepts that are related to integers and squares were also different. 
Both speakers jointly designed a passage on "positive integers as a sum of squares." Each took turns reading the passage to control for recognition errors associated with context, auditory, and timing. The same recording machine was used by both speakers to control for hesitation and voice quality on the mathematical terms and concepts.

\section{Data Management}

Each group took the quiz after being exposed to the material only once. Both sets of students were comparable in education and were randomly selected from the same initial group. Each set independently listened to either the non-Ugandan or native Ugandan instructor's pre-recorded mathematics passage in English, after which they answered questions from a quiz on the topic. Response Forms were marked $\mathrm{L}_{1}$, and $\mathrm{L}_{2}$. Forms marked $\mathrm{L}_{1}$ were tagged to a Ugandan English speaker accent and $\mathrm{L}_{2}$ to the non-Ugandan with foreign accent. None of the participants had prior access to the passage or the quiz.

\section{Data analyses}

Correct responses indicate comprehension of the mathematical terms and concepts. Questions 1 to 3 dealt with basic introduction of the squares. Questions 4-7 required additional comprehension and processing by the students to answer the questions. For this, the students needed to understand the speaker's oral explanation of how to express numbers as sums of squares. These questions directly tested whether students' understanding of the mathematical meanings were affected by the pronunciation of mathematical terms.

\section{$\underline{\text { Results: }}$}

The results are presented in Table 1 as shown below.

Table 1: Students Quiz Responses

\begin{tabular}{lll}
\hline \multirow{2}{*}{ Quiz Question } & \multicolumn{2}{l}{ SPEAKER } \\
\cline { 2 - 3 } & L1 & L2 \\
\hline & $(\mathrm{a}, \mathrm{b}, \mathrm{c})$ & $(\mathrm{a}, \mathrm{b}, \mathrm{c})$ \\
Q1 & $(9,0,0)$ & $(9,0,0)$ \\
Q2 & $(9,0,0)$ & $(9,0,0)$ \\
Q3 & $(8,0,1)$ & $(8,0,1)$ \\
\hline
\end{tabular}




\begin{tabular}{lll}
\hline Q4 & $(6,2,1)$ & $(1,5,3)$ \\
Q5 & $(2,1,6)$ & $(0,3,6)$ \\
Q6 & $(4,4,1)$ & $(2,3,4)$ \\
Q7 & $(6,0,3)$ & $(4,0,5)$
\end{tabular}

Number of students to each 9
speaker

9

\section{KEY:}

$\mathbf{L}_{\mathbf{1}}$ : stands for local speaker $\mathbf{L}_{2}$ : stands for foreign speaker

The ordered triple $(\mathrm{a}, \mathrm{b}, \mathrm{c})$ indicates the frequency of answer where
- $\mathbf{a}=$ correct answers

- $\mathbf{b}=$ correct answers but based on wrong reasons

- $\mathbf{c}=$ wrong answers

\section{Discussion}

For Q 1, 2, and 3: The first three questions were meant to be straightforward. The answers were given in the reading itself, except that the answer to question 3 involved a concept. The answer " 4 " to question 3 was correct and it was not necessary to write "maximum" in words, hence, if a student wrote "requires 4 squares" it was an incorrect answer. Every positive integer does not "require" four squares in its representation as the sum of the squares.

With regard to Q4, the answer with correct reasoning must have three components: first, 37 is a prime, second, the remainder of 1 is obtained after dividing by 4 , and then recognizing the fact that only two squares are needed and are guaranteed by the result in the reading.

For Q5, the answer with correct reasoning must have four components: first, 43 is a prime, second, the remainder of 3 is obtained after dividing by 4 , and then recognizing the fact that at least three squares were needed but are not guaranteed by the result in the reading. So, students had to actually find a correct/required representation. (e.g., $43=5^{2}+3^{2}+3^{2}$ ). 
In Q6, here students should not have taken the remainder as it was not a prime and they should have indicated that, at most, 4 squares were needed, and they should have checked and recognized that they can do better: $38=6^{2}+1^{2}+1^{2}$.

For Q7, our expected answer was 1 square, as $25=5^{2}$, and only one square was needed.

Though our data set was small, we saw clear differences between the two groups. When exposed to a teacher with a non-native accent, the students' answers to the quiz clearly show their inability to understanding the instructor's oral explanation of mathematical terms and concepts. This, however, was not exhibited by the second set of students who heard exactly the same material presented in a native tone and accent. For example, two students understood that the remainder is 1 , then they need two squares, and if the remainder is 3, they may need 3 or 4 squares, but these students assumed the answer is remainder +1 squares. Then instead of the remainder, they used either the quotient or actual answer in decimals and rounded it up to give the answer to the number of squares needed for various numbers asked on the test, namely 37,43 , and 38 .

\section{Conclusion}

Given that student will often interact with teachers of different nationalities and with different accents in the context of globalization and internationalization of education, our study flags the possibility of a resultant barrier that this might pose to the student's learning and, specifically, and specifically in our case, the challenge to their understanding of mathematical terms and concepts. This, however, can be overcome by acknowledging the problem and working for a solution. Foreign teachers may have to do more preparation than previously expected to overcome the communication hurdles. For example, to be an effective teacher, they may have to provide more written explanations and do more board work than their native counterparts. In addition, this difficulty also likely arises in the opposite direction. Universities and schools that are actively recruiting foreign students may find that foreign students have difficulty adjusting to instructions given due to difficulties with native accent. These students may require extra or alternative methods of instruction in order for them to be successful.

To combat the possible barrier to learning due to foreign accent, local talents must be encouraged to become teachers and local communities must endeavour to retain them by according them proper pay and respect. Even if a foreign candidate may be slightly more qualified in terms of 
certification, nonetheless, universities hiring local candidates may be better for the promotion of students' learning outcomes and in the long run is likely to be more cost-efficient.

In conclusion, although diversity in education is considered to be generally positive in terms of the exposure and experiences it brings, it can also bring hurdles. Learners as well as instructors therefore need to expend effort to ensure that a difference in communication does not hinder learning.

\section{Disclaimer}

This paper is based on the experiment conducted by the authors; one of them an instructor on fellowship appointment from the US and the other two, host country instructors, during our interactions with students on a Science with Education training. The opinions, findings, and conclusions or recommendations expressed in this study are those of the authors and do not reflect the views of Mbarara University of Science and Technology.

\section{$\underline{\text { References }}$}

Campbell, J., \& Li, M. (2008). Asian students' voices: An empirical study of Asian students' learning experiences at a New Zealand University. Journal of Studies in International Education, 12,375-396. Doi:10.1177/1028315307299422.

Gurel Cennetkusu, N., Koc, H., \& Teker Kozcaz, E. (2020). An Experimental Study on Incidental Voca-bulary Acquisition: Incidental Vocabulary Acquisition in Foreign Language by Visual Literary Quotations. Open Access Library Journal, 7:e6176. https://doi.org/10.4236/oalib.1106176.

Hou, Y. A. (2018). A Study of the Role of Strategy in Foreign Language Learning. Open Journal of Modern Linguistics, 8, 151-175. https://doi.org/10.4236/ojm.2018.85016.

Keene, E. O. \& Zimmermann, S. (1997). Mosaic of Thought: The power of comprehension strategy instruction. Heinemann.

Kolapo, F. J. (2009). Immigrant Academics and Cultural Challenges in a Global Environment. Amherst, NY: Cambria Press.

Lippi-Green, R. (2012). English with an accent: Language, ideology, and discrimination in the United States, NewYork: Routlege. 354page. ISBN-100415559103/ IBSN-13:9780415559102.

Salomon, G. (1983). The differential investment of mental effort in learning from different sources. Education Psychologist, 18, 42-50. 
Sicat, R. M. (2011). Foreign students' cultural adjustment and coping strategies. International Proceedings of Economics Development \& Research, 5, 338-341. Available at http://www.ipedr.com.

Smith, F. (1994). Understanding reading: A Psycholinguistic analysis of reading and learning to read. Hillside, NJ: Lawrence Erlbaum Associates.

Taussky, O. (1970). Sums of Squares. Available at https://www.maa.org/sites/default/files/pdf/upload_library/22/Ford/OlgaTaussky.pdf

Republic of Uganda (1995). Constitution of the Republic of Uganda, 1995.

Park, E., Klieve, H., Tsurutani, C., \& Harte, W. (2017). International Students' accented English, Communication difficulties and developed Strategies, Journal of Cogent Education, 4(1).

Valles, B. (2015). "The Impact of Accented English on Speech Comprehension" (2015). Open Access Theses \& Dissertations, 1175. Available at https://digitalcommons.utep.edu/open_etd/1175. 